\title{
ABOUT THE ANTHROPOMORPHIC ROBOTS
}

\author{
Florian Ion Petrescu ${ }^{1}$ \\ Relly Victoria Petrescu ${ }^{2}$
}

\begin{abstract}
The paper presents an original geometrical and kinematic method for the study of geometry and determining positions of a MP-3R structure of the anthropomorphic robots. It presents shortly the MP-3R direct and inverse kinematics, the inverse kinematics being solved by an original exactly method. One presents shortly an original method to solve the robot inverse kinematics exemplified at the 3R-Robots (MP-3R). The system which must be solved has three equations and three independent parameters to determine. Constructive basis is represented by a robot with three degrees of freedom (a robot with three axes of rotation). If we study (analyze) an anthropomorphic robot with three axes of rotation (which represents the main movements, absolutely necessary), we already have a base system, on which we can then add other movements (secondary, additional). Calculations were arranged and in the matrix form. The most commonly used serial structures over the last 20 or 30 years are those of type $3 R, 4 R, 5 R, 6 R$, having as constituents essential basic kinematic chain $3 R$, robot anthropomorphic (RRR), where main rotation around a vertical axis, causes the construction. It can thus passes from the study spatial movement, which is more difficult, to the study motion plane, basic movement, for all the robots and fillers serial movements of rotation. Moving flat, horizontal or vertical, shall be undertaken far more easily than the spatial integration with the convenience simple in the space of which it is part. We will exemplify the basic structure existing in a few serial platforms of rotation, these being the most generalized (more widespread) at the present time. In this work will be pursued and the direct and inverse kinematics of these mechanical systems. It can make the transition from $3 R$ systems-level 2R and vice versa.
\end{abstract}

Keywords: Anthropomorphic robots, direct kinematics, inverse kinematics, 3R systems, 2R systems

\footnotetext{
${ }^{1}$ Bucharest Polytechnic University, Romania. Email : petrescuflorian@yahoo.com

${ }^{2}$ Bucharest Polytechnic University, Romania . Email: petrescuvictoria@yahoo.com
} 


\section{INTRODUCTION}

Moving mechanical structures (Garcia E., 2007; Garcia M., 2013; He, 2013; Reddy, 2012) are used increasingly in almost all vital sectors of humanity (Tang, 2013; Tong, 2013). The robots are able to process integrated circuits sizes micro and nano, on which the man they can be seen even with electron microscopy (Lee, 2013). Dyeing parts in toxic environments, working in chemical and radioactive environments, or at depths and pressures at the bottom of huge oceans, or even cosmic space conquest and visiting exo-planets, are now possible, and were turned into from the dream in reality, because mechanical platforms sequential gearbox (Dong, 2013; Wang, 2013).

The man will be able to carry out its mission supreme, conqueror of new galaxies, because mechanical systems sequential gearbox (Perumaal, 2013).

Robots (Flavio de Melo, 2012) were developed and diversified, different aspects, but to-day, they start to be directed on two major categories: systems serial and parallel systems (Padula, 2013).

Parallel systems (Cao, 2013; Lin, 2013; Tabaković, 2012) are more solid, but more difficult to designed and handled, which serial systems were those which have developed the most.

Serial systems and they have different constructive diagrams, but over the last 30 years have been channelled on anthropomorphic structures (Reddy, 2012).

These structures are made up of simple components and couplers for rotation.

Their great advantage is fast movements, good dynamics, a high accuracy, a construction of simple modules STAS, economy of materials, low cost, and high reliability.

One disadvantage of less accurate has been removed because of stepper motors.
Compared with parallel systems (more solid but more cumbersome) serial systems may pose, and the disadvantage of stability something lower.

This disadvantage begins to be exceeded today in smart mode, through the construction of serial systems made up of elements doubled (in parallel).

This last invention, will lead to the strengthening of serial systems, and to their consolidation like the indisputable leader in diversity of mechatronics and robotic systems.

This work starts from a main idea, to study these systems on a single model, $3 \mathrm{R}$, which has finally main movements lying on a single plane model, $2 \mathrm{R}$.

\section{A BASIC 3R STRUCTURE OF MOVING (SERIAL) MECHANICAL SYSTEMS}

The most commonly used serial structures over the last 20 or 30 years are those of type $3 R, 4 R, 5 R, 6 R$, having as constituents essential basic kinematic chain 3R, robot anthropomorphic (RRR), where main rotation around a vertical axis, causes the construction (Petrescu, 2012).

There are then a basic kinematic chain which has two revolutions 'bokeh' (two actuators, i.e. two motors) who work permanently in one plane, and immediately after main support which supports and rotates vertically complete assembly (Petrescu, 2011).

This basic structure (Tang, 2013), $3 \mathrm{R}$, a meet me at all robots serial manufactured on the principle of rotations. Vertical Bracket is was the same, but the drive train as follows, with the two turns situated in a plane can be positioned vertically (most often; the robots anthropomorphic, fig. 1b), or horizontally (case robots scale, fig. 1a). 

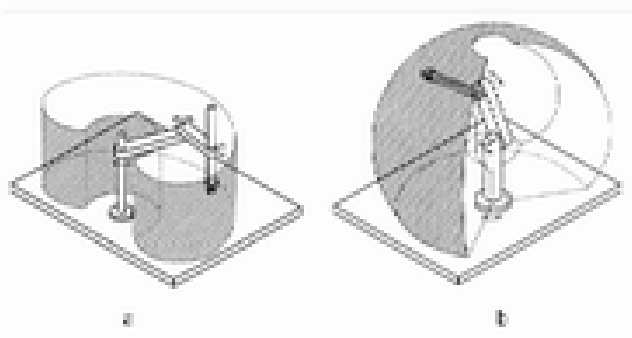

Fig. 1. The basic structures $3 R$ (a-scale structure ; $b$-anthropomorphic structure)

It can thus passes from the study spatial movement, which is more difficult, to the study motion plane, basic movement, for all the robots and fillers serial movements of rotation.

Moving flat, horizontal or vertical, shall be undertaken far more easily than the spatial integration with the convenience simple in the space of which it is part (Garcia, 2007).

We will exemplify the basic structure existing in a few serial platforms of rotation, these being the most generalized (more widespread) at the present time. On this basic model (3R) have developed further robots 6R (He, 2013) today (fig. 2 , rely only on revolutions using actuator that only electric motors, compact); they have a hardness greater penetration while maintaining the flexibility and models $3 \mathrm{R}, 4 \mathrm{R}$ and $5 \mathrm{R}$.

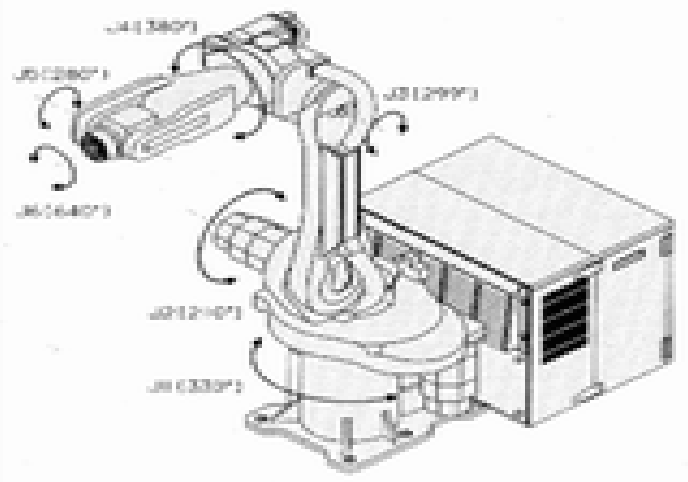

Fig. 2. Structure 6R (anthropomorphic structure)
Almost all major companies come today with models 6R (which they improve continuously).

Why they have imposed today these models of robots (after tens of years of diversity was the word of order); may and of the need for standardization, or to find a common solution, after a huge portion (however are not yet the only robots use of category serial, but they also have the widest spread).

The six turns (full elimination of translations, who bring many disadvantages due to coupler $T$ itself) are operating easier, faster, with higher yield, more reliable, more compact and more secure; basic revolutions, remain all first three, the other three turns (additional) having the role of position may well end device, the end effector. Results and that the baseline study (required) it is still not for a $3 R$.

This can be seen in the latest models of the various firms producing robots (fig. 3, Kawasaki, Romat, Fanuc, Motoman, Kuka, etc). And the structures used inside cells sequential gearbox are constructed generally in a similar way.

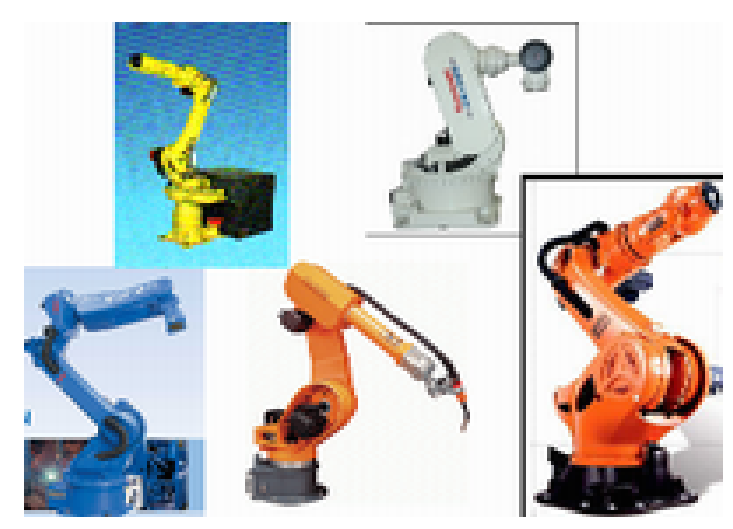

Fig. 3. Miscellaneous modern structures 6R (anthropomorphic robots)

In figure 4 is illustrated geometrocinematic a structure of basic $3 R$.

Starting from this platform may be studied by addition any other scheme, n$\mathrm{R}$ modern. 
The platform (system) in figure 4, has three degree of mobility, which can be realized by three actuators (electric motors).

First electric motor drives your whole system in a rotating around a vertical spindle $\mathrm{O}_{0} \mathrm{z}_{0}$. Engine (actuator) number 1 , is mounted on the fixed (frame, 0 ) and causes mobile element 1 in a rotating around a vertical axis (The mobile element 1, then all the other elements (components) of the system).

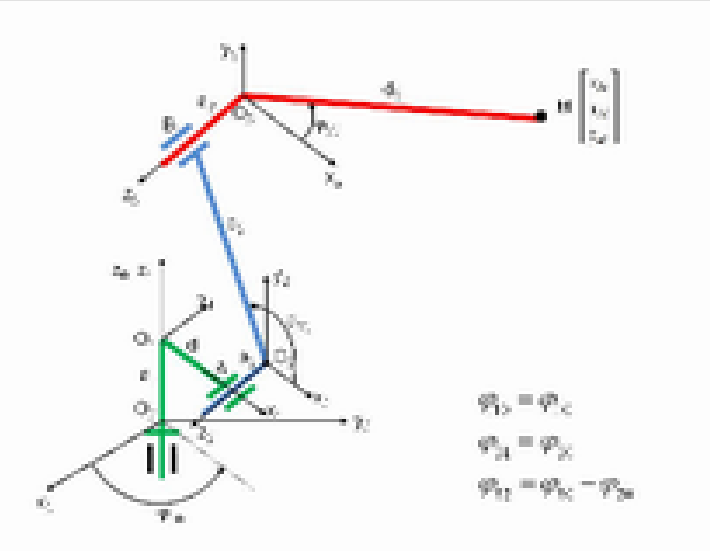

Fig. 4. Layout geometro-cinematic of a modern structures 3R (anthropomorphic)

\section{1 (3R) Direct kinematics}

In direct kinematics (Petrescu, 20092013) are known the rotation parameters, $\varphi_{10}, \varphi_{20}, \varphi_{30}$, and must be determined the parameters of the point M. Relations of calculation used (system 1) are the following.

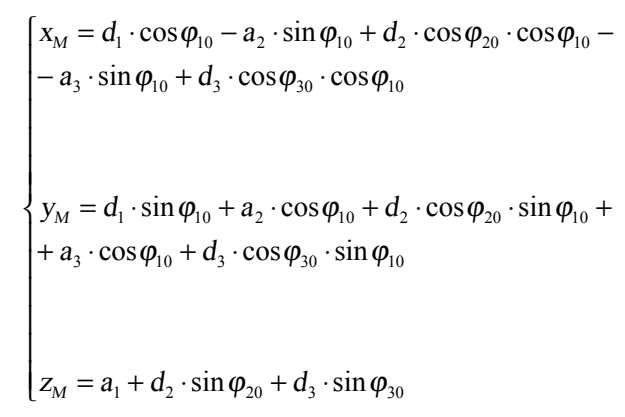

\section{$2.2(3 R)$ Inverse kinematics}

In inverse kinematics (Liu, 2013) are known the parameters of the point $\mathrm{M}$, and must be determined the rotation parameters, $\varphi_{10}, \varphi_{20}, \varphi_{30}$. Relations of calculation used (system 2-4) are the following.

$$
\left\{\begin{array}{l}
\cos \varphi_{10}=\frac{\left(a_{2}+a_{3}\right) \cdot y_{M} \pm x_{M} \cdot \sqrt{x_{M}^{2}+y_{M}^{2}-\left(a_{2}+a_{3}\right)^{2}}}{x_{M}^{2}+y_{M}^{2}} \\
\sin \varphi_{10}=\frac{-\left(a_{2}+a_{3}\right) \cdot x_{M} \pm y_{M} \cdot \sqrt{x_{M}^{2}+y_{M}^{2}-\left(a_{2}+a_{3}\right)^{2}}}{x_{M}^{2}+y_{M}^{2}}
\end{array}\right.
$$

$$
\left\{\begin{array}{l}
C_{1}=x_{M} \cdot \cos \varphi_{10}+y_{M} \cdot \sin \varphi_{10}-d_{1} \\
C_{2}=z_{M}-a_{1} \\
k=C_{1}^{2}+C_{2}^{2}+d_{2}^{2}-d_{3}^{2}
\end{array}\right.
$$

$$
\left\{\begin{array}{l}
\cos \varphi_{20}=\frac{k \cdot C_{1} \pm C_{2} \cdot \sqrt{4 \cdot C_{1}^{2} \cdot d_{2}^{2}+4 \cdot C_{2}^{2} \cdot d_{2}^{2}-k^{2}}}{2 \cdot\left(C_{1}^{2}+C_{2}^{2}\right) \cdot d_{2}} \\
\sin \varphi_{20}=\frac{k \cdot C_{2}{ }^{*} C_{1} \cdot \sqrt{4 \cdot C_{1}^{2} \cdot d_{2}^{2}+4 \cdot C_{2}^{2} \cdot d_{2}^{2}-k^{2}}}{2 \cdot\left(C_{1}^{2}+C_{2}^{2}\right) \cdot d_{2}} \\
\varphi_{20}=\operatorname{semn}\left(\sin \varphi_{20}\right) \cdot \arccos \left(\cos \varphi_{20}\right) \\
\cos \varphi_{30}=\frac{C_{1}-d_{2} \cdot \cos \varphi_{20}}{d_{3}} \\
\sin \varphi_{30}=\frac{C_{2}-d_{2} \cdot \sin \varphi_{20}}{d_{3}} \\
\varphi_{30}=\operatorname{semn}\left(\sin \varphi_{30}\right) \cdot \arccos \left(\cos \varphi_{30}\right)
\end{array}\right.
$$

\section{A BASIC 2R STRUCTURE OF MOVING (SERIAL) MECHANICAL SYSTEMS}

In figure 5 can be monitored kinematic chain schematic plan 23 opened. If de-couple the rotating movement around the vertical axis $3 \mathrm{R}$ system (Fig. 4) becomes a system plan 2R (Fig. 5). 


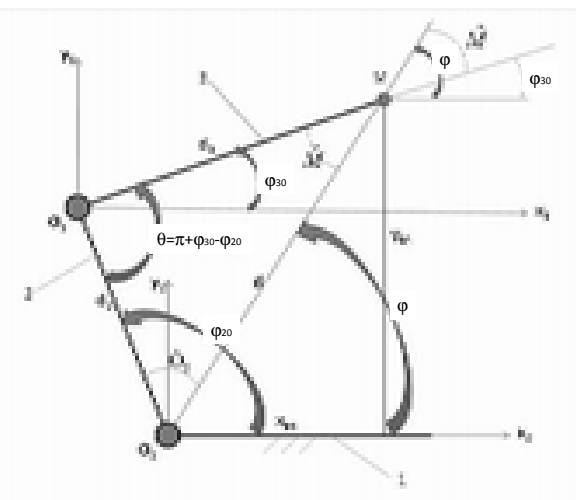

Fig. 5. Cinematic schematic kinematic chain plan 2-3 linked to item 1 considered fixed

\section{1 (2R) Direct kinematics}

In the direct kinematics parameters (Wang, 2013) are known the parameters cinematic $\varphi_{20}$ and $\varphi_{30}$ and must be determined by analytical calculation parameters $\mathrm{x}_{\mathrm{M}}$ and $\mathrm{y}_{\mathrm{M}}$, which represents the co-ordinates of scaling point $\mathrm{M}$ (endeffector $\mathrm{M}$ ). Designing vectors $\mathrm{d}_{2}+$ $\mathrm{d}_{3}$ on cartesian axis system considered fixed, $x O y$, identical to $\mathrm{x}_{2} \mathrm{O}_{2} \mathrm{y}_{2}$. Obtain the equation scaling (5) (Wen, 2012).

$$
\left\{\begin{array}{l}
x_{2 M} \equiv x_{M}=x_{O_{3}}+x_{3 M}= \\
=d_{2} \cdot \cos \varphi_{20}+d_{3} \cdot \cos \varphi_{30}=d \cdot \cos \varphi \\
y_{2 M} \equiv y_{M}=y_{O_{3}}+y_{3 M}= \\
=d_{2} \cdot \sin \varphi_{20}+d_{3} \cdot \sin \varphi_{30}=d \cdot \sin \varphi
\end{array}\right.
$$

After you determine the co-ordinates of Cartesian point $\mathrm{M}$ using data relations of the system (5), may be obtained immediately and parameters using angle relationships established within the framework of the system (6).

$$
\left\{\begin{array}{l}
d^{2}=x_{M}^{2}+y_{M}^{2} ; d=\sqrt{x_{M}^{2}+y_{M}^{2}} \\
\cos \varphi=\frac{x_{M}}{d}=\frac{x_{M}}{\sqrt{x_{M}^{2}+y_{M}^{2}}} \\
\sin \varphi=\frac{y_{M}}{d}=\frac{y_{M}}{\sqrt{x_{M}^{2}+y_{M}^{2}}} \\
\varphi=\operatorname{sign}(\sin \varphi) \cdot \arccos (\cos \varphi)
\end{array}\right.
$$

The system (5) is being written brief in the form (7).

$$
\left\{\begin{array}{l}
x_{M}=d_{2} \cdot \cos \varphi_{20}+d_{3} \cdot \cos \varphi_{30}= \\
=d_{2} \cdot \cos \varphi_{20}+d_{3} \cdot \cos \left(\theta+\varphi_{20}-\pi\right) \\
y_{M}=d_{2} \cdot \sin \varphi_{20}+d_{3} \cdot \sin \varphi_{30}= \\
=d_{2} \cdot \sin \varphi_{20}+d_{3} \cdot \sin \left(\theta+\varphi_{20}-\pi\right)
\end{array}\right.
$$

\section{2 (2R) Inverse kinematics}

First, it determines the intermediary parameters, $d$ and $\varphi$ with relations (8).

$$
\left\{\begin{array}{l}
d^{2}=x_{M}^{2}+y_{M}^{2} ; \quad d=\sqrt{x_{M}^{2}+y_{M}^{2}} \\
\cos \varphi=\frac{x_{M}}{d}=\frac{x_{M}}{\sqrt{x_{M}^{2}+y_{M}^{2}}} ; \\
\sin \varphi=\frac{y_{M}}{d}=\frac{y_{M}}{\sqrt{x_{M}^{2}+y_{M}^{2}}} \\
\varphi=\operatorname{sign}(\sin \varphi) \cdot \arccos (\cos \varphi)
\end{array}\right.
$$

In some triangle $\mathrm{O}_{2} \mathrm{O}_{3} \mathrm{M}$ known lengths of the three sides, $\mathrm{d}_{2}, \mathrm{~d}_{3}$ (constant) and $d$ (variable), so that may be determined according to sides lengths all other elements of triangle, and more specifically its angles, trigonometric functions and their (us great interest in sin and $\cos$ ).

For the purpose of determining angles can be used various methods (trigonometric, geometric, etc.), of which will be presented below one of them (as the most representative): trigonometric method.

Shall I write the equations of positions scaling (9).

$$
\left\{\begin{array}{l}
d_{2} \cdot \cos \varphi_{20}+d_{3} \cdot \cos \varphi_{30}=x_{M} \\
d_{2} \cdot \sin \varphi_{20}+d_{3} \cdot \sin \varphi_{30}=y_{M} \\
\cos ^{2} \varphi_{20}+\sin ^{2} \varphi_{20}=1 \\
\cos ^{2} \varphi_{30}+\sin ^{2} \varphi_{30}=1
\end{array}\right.
$$

These two equations problem scaling, trigonometric, with two unknown $\left(\varphi_{20}\right.$ and $\left.\varphi_{30}\right)$ is that they 
are more often met and slowly exceed (are equations trigonometric, transcendental equations, where unknown does not appear directly $\varphi_{20}$ but also in the form $\cos \varphi_{20}$ and $\sin \varphi_{20}$, so that in reality within the framework of the two equations trigonometric don't have two unknown but four: $\cos \varphi_{20}$, $\sin \varphi_{20}, \cos \varphi_{30}$ and $\left.\sin \varphi_{30}\right)$. To resolve system we need two more equations, so that in the system (9) has been added two more equations trigonometric, more exactly the equations trigonometric of base "gold" as they say, for the angle $\varphi_{20}$ and separately for the angle $\varphi_{30}$.

With a view to resolving the two equations of the system (9) shall be written in the form (10).

$$
\left\{\begin{array}{l}
d_{2} \cdot \cos \varphi_{20}-x_{M}=-d_{3} \cdot \cos \varphi_{30} \\
d_{2} \cdot \sin \varphi_{20}-y_{M}=-d_{3} \cdot \sin \varphi_{30}
\end{array}\right.
$$

Each equation of the system (10) rose square, after which summed up both equation (raised square) and obtain the equation of the form (11).

$$
\begin{aligned}
& d_{2}^{2} \cdot\left(\cos ^{2} \varphi_{20}+\sin ^{2} \varphi_{20}\right)+x_{M}^{2}+y_{M}^{2}- \\
& -2 \cdot d_{2} \cdot x_{M} \cdot \cos \varphi_{20}-2 \cdot d_{2} \cdot y_{M} . \\
& \cdot \sin \varphi_{20}=d_{3}^{2} \cdot\left(\cos ^{2} \varphi_{30}+\sin ^{2} \varphi_{30}\right)
\end{aligned}
$$

Now is the time to use the two "equation of gold" trigonometric system written at the end (9), whereby the equation (11) becomes simplified form (12).

$$
\begin{aligned}
& d_{2}^{2}+x_{M}^{2}+y_{M}^{2}-2 \cdot d_{2} \cdot x_{M} \cdot \cos \varphi_{20}- \\
& -2 \cdot d_{2} \cdot y_{M} \cdot \sin \varphi_{20}=d_{3}^{2}
\end{aligned}
$$

$$
\begin{aligned}
& d_{2}^{2}-d_{3}^{2}+x_{M}^{2}+y_{M}^{2}= \\
& =2 \cdot d_{2} \cdot\left(x_{M} \cdot \cos \varphi_{20}+y_{M} \cdot \sin \varphi_{20}\right)
\end{aligned}
$$

Divide the equation (13) with $2 \cdot d_{2}$ and will result in an new form (14).

$$
\begin{aligned}
& x_{M} \cdot \cos \varphi_{20}+y_{M} \cdot \sin \varphi_{20}= \\
& =\frac{d_{2}^{2}-d_{3}^{2}+x_{M}^{2}+y_{M}^{2}}{2 \cdot d_{2}}
\end{aligned}
$$

As shown in figure 5 shall be deducted and the relationship (15) which may also appears in the system (8).

$$
x_{M}^{2}+y_{M}^{2}=d^{2}
$$

Enter the expression (15) to (14) and multiply the fraction from the right with $\mathrm{d}$, so that the expression (14) takes the form convenient (16).

$$
x_{M} \cdot \cos \varphi_{20}+y_{M} \cdot \sin \varphi_{20}=\frac{d_{2}^{2}+d^{2}-d_{3}^{2}}{2 \cdot d_{2} \cdot d} \cdot d
$$

Now it's time of introduction of expression cosine of $\mathrm{O}_{2}$ angle, depending on the sides of the some triangle $\mathrm{O}_{2} \mathrm{O}_{3} \mathrm{M}$ (Fig. 5) (17).

$$
\cos \hat{O}_{2}=\frac{d_{2}^{2}+d^{2}-d_{3}^{2}}{2 \cdot d_{2} \cdot d}
$$

With the relation (17) the equation (16) becomes simplified form (18).

Arrange the terms of this equation (12) in the form most convenient (13).

$$
\begin{aligned}
& x_{M} \cdot \cos \varphi_{20}-d \cdot \cos \hat{O}_{2}= \\
& =-y_{M} \cdot \sin \varphi_{20}
\end{aligned}
$$


We want to eliminate $\sin \varphi_{20}$, from which I isolate the term in sin, and got to his feet to square the equation (18), so that by using the equation gold (trigonometric) for the angle $\varphi_{20}$ to transform $\sin$ in cos, equation become one of the second degree in $\cos \varphi_{20}$. After raising square (18) takes shape (19).

$$
\begin{aligned}
& x_{M}^{2} \cdot \cos ^{2} \varphi_{20}+d^{2} \cdot \cos ^{2} \hat{O}_{2}- \\
& -2 \cdot d \cdot x_{M} \cdot \cos \hat{O}_{2} \cdot \cos \varphi_{20}= \\
& =y_{M}^{2} \cdot \sin ^{2} \varphi_{20}
\end{aligned}
$$

Using formula of gold, the expression (19) takes shape (20) which is arranges convenient by grouping terms, and bringing it to the form (21).

$$
\begin{aligned}
& x_{M}^{2} \cdot \cos ^{2} \varphi_{20}+d^{2} \cdot \cos ^{2} \hat{O}_{2}- \\
& -2 \cdot d \cdot x_{M} \cdot \cos \hat{O}_{2} \cdot \cos \varphi_{20}= \\
& =y_{M}^{2}-y_{M}^{2} \cdot \cos ^{2} \varphi_{20} \\
& \left(x_{M}^{2}+y_{M}^{2}\right) \cdot \cos ^{2} \varphi_{20}- \\
& -2 \cdot d \cdot x_{M} \cdot \cos \hat{O}_{2} \cdot \cos \varphi_{20}- \\
& -\left(y_{M}^{2}-d^{2} \cdot \cos ^{2} \hat{O}_{2}\right)=0
\end{aligned}
$$

Discriminant of the equation (21) of second degree in cos obtained shall be calculated according to the relation (22).

$$
\left\{\begin{array}{l}
\Delta=d^{2} \cdot x_{M}^{2} \cdot \cos ^{2} \hat{O}_{2}+ \\
+d^{2} \cdot\left(y_{M}^{2}-d^{2} \cdot \cos ^{2} \hat{O}_{2}\right)= \\
=d^{2} \cdot\left(x_{M}^{2} \cdot \cos ^{2} \hat{O}_{2}+y_{M}^{2}-\right. \\
\left.-d^{2} \cdot \cos ^{2} \hat{O}_{2}\right)= \\
=d^{2} \cdot\left(y_{M}^{2}-y_{M}^{2} \cdot \cos ^{2} \hat{O}_{2}\right)= \\
=d^{2} \cdot y_{M}^{2} \cdot\left(1-\cos ^{2} \hat{O}_{2}\right)= \\
=d^{2} \cdot y_{M}^{2} \cdot \sin ^{2} \hat{O}_{2}
\end{array}\right.
$$

Radical from the second order of the discriminant is expressed as (23).

$$
\begin{aligned}
& R=\sqrt{\Delta}=\sqrt{d^{2} \cdot y_{M}^{2} \cdot \sin ^{2} \hat{O}_{2}}= \\
& =d \cdot y_{M} \cdot \sin \hat{O}_{2}
\end{aligned}
$$

Equation solutions (20) second degree in cos shall be written in the form (24).

$$
\left\{\begin{array}{l}
\cos \varphi_{20_{1,2}}= \\
=\frac{d \cdot x_{M} \cdot \cos \hat{O}_{2}^{*} d \cdot y_{M} \cdot \sin \hat{O}_{2}}{d^{2}}= \\
=\frac{x_{M} \cdot \cos \hat{O}_{2}^{*} y_{M} \cdot \sin \hat{O}_{2}}{d}= \\
=\frac{x_{M}}{d} \cdot \cos \hat{O}_{2}^{*} \frac{y_{M}}{d} \cdot \sin \hat{O}_{2}
\end{array}\right.
$$

In the solutions (24) are replaced the reports with the corresponding functions trigonometric of the angle $\varphi$, expressions (24) mellowed form (25).

$$
\left\{\begin{array}{l}
\cos \varphi_{20_{1,2}}= \\
=\frac{x_{M}}{d} \cdot \cos \hat{O}_{2}^{*} \frac{y_{M}}{d} \cdot \sin \hat{O}_{2}= \\
=\cos \varphi \cdot \cos \hat{O}_{2}^{*} \sin \varphi \cdot \sin \hat{O}_{2}= \\
=\cos \left(\varphi \pm \hat{O}_{2}\right) \\
\cos \varphi_{20}=\cos \left(\varphi \pm \hat{O}_{2}\right)
\end{array}\right.
$$


Now we're going back to the equation (18) that is ordered in the form (26), in order to solve them in sin. The equation (26) rose square and through the use of gold trigonometric equation of angle $\varphi_{20}$, is obtained form (27).

$$
\begin{aligned}
& x_{M} \cdot \cos \varphi_{20}= \\
& =d \cdot \cos \hat{O}_{2}-y_{M} \cdot \sin \varphi_{20} \\
& \left\{\begin{array}{l}
x_{M}^{2} \cdot \cos ^{2} \varphi_{20}=d^{2} \cdot \cos ^{2} \hat{O}_{2}+ \\
+y_{M}^{2} \cdot \sin ^{2} \varphi_{20}- \\
-2 \cdot y_{M} \cdot d \cdot \cos \hat{O}_{2} \cdot \sin \varphi_{20} \\
x_{M}^{2}-x_{M}^{2} \cdot \sin ^{2} \varphi_{20}= \\
=d^{2} \cdot \cos ^{2} \hat{O}_{2}+y_{M}^{2} \cdot \sin { }^{2} \varphi_{20}- \\
-2 \cdot y_{M} \cdot d \cdot \cos \hat{O}_{2} \cdot \sin \varphi_{20} \\
\left(x_{M}^{2}+y_{M}^{2}\right) \cdot \sin ^{2} \varphi_{20}- \\
-2 \cdot y_{M} \cdot d \cdot \cos \hat{O}_{2} \cdot \sin \varphi_{20}- \\
-\left(x_{M}^{2}-d^{2} \cdot \cos \hat{O}_{2}\right)=0 \\
d^{2} \cdot \sin ^{2} \varphi_{20}- \\
-2 \cdot y_{M} \cdot d \cdot \cos \hat{O}_{2} \cdot \sin \varphi_{20}- \\
-\left(x_{M}^{2}-d^{2} \cdot \cos \hat{O}_{2}\right)=0
\end{array}\right.
\end{aligned}
$$

$$
\left\{\begin{array}{l}
\sin \varphi_{20}= \\
=\frac{y_{M} \cdot d \cdot \cos \hat{O}_{2} \pm x_{M} \cdot d \cdot \sin \hat{O}_{2}}{d^{2}}= \\
=\frac{y_{M} \cdot \cos \hat{O}_{2} \pm x_{M} \cdot \sin \hat{O}_{2}}{d}= \\
=\frac{y_{M}}{d} \cdot \cos \hat{O}_{2} \pm \frac{x_{M}}{d} \cdot \sin \hat{O}_{2}= \\
=\sin \varphi \cdot \cos \hat{O}_{2} \pm \cos \varphi \cdot \sin \hat{O}_{2}= \\
=\sin \left(\varphi \pm \hat{O}_{2}\right) \\
\sin \varphi_{20}=\sin \left(\varphi \pm \hat{O}_{2}\right)
\end{array}\right.
$$

Have been obtained relations (30), of which shall be deducted the basic relationship (31).

$$
\begin{array}{r}
\left\{\begin{array}{l}
\cos \varphi_{20}=\cos \left(\varphi \pm \hat{O}_{2}\right) \\
\sin \varphi_{20}=\sin \left(\varphi \pm \hat{O}_{2}\right)
\end{array}\right. \\
\varphi_{20}=\varphi \pm \hat{O}_{2}
\end{array}
$$

Repeat the procedure and for determining $\varphi_{30}$ angle, starting again from the system (9), in which the first two equations transcedental are rewritten in the form (32), with a view to eliminating $\varphi_{20}$ angle at this time.

Discriminant of the equation (27) of second degree in cos takes the form (28).

$$
\left\{\begin{array}{l}
\Delta=y_{M}^{2} \cdot d^{2} \cdot \cos ^{2} \hat{O}_{2}+ \\
+d^{2} \cdot\left(x_{M}^{2}-d^{2} \cdot \cos ^{2} \hat{O}_{2}\right)= \\
=d^{2} \cdot\left(x_{M}^{2}+y_{M}^{2} \cdot \cos ^{2} \hat{O}_{2}-\right. \\
\left.-x_{M}^{2} \cdot \cos ^{2} \hat{O}_{2}-y_{M}^{2} \cdot \cos ^{2} \hat{O}_{2}\right)= \\
=d^{2} \cdot\left(x_{M}^{2}-x_{M}^{2} \cdot \cos ^{2} \hat{O}_{2}\right)= \\
=d^{2} \cdot x_{M}^{2} \cdot \sin ^{2} \hat{O}_{2}
\end{array}\right.
$$

Equation Solutions (27) shall be written in the form (29).

$$
\begin{gathered}
\left\{\begin{array}{l}
d_{2} \cdot \cos \varphi_{20}+d_{3} \cdot \cos \varphi_{30}=x_{M} \\
d_{2} \cdot \sin \varphi_{20}+d_{3} \cdot \sin \varphi_{30}=y_{M} \\
\cos ^{2} \varphi_{20}+\sin ^{2} \varphi_{20}=1 \\
\cos ^{2} \varphi_{30}+\sin ^{2} \varphi_{30}=1
\end{array}\right. \\
\left\{\begin{array}{l}
d_{2} \cdot \cos \varphi_{20}=x_{M}-d_{3} \cdot \cos \varphi_{30} \\
d_{2} \cdot \sin \varphi_{20}=y_{M}-d_{3} \cdot \sin \varphi_{30}
\end{array}\right.
\end{gathered}
$$

In the end we get the system 33, having regard the solution 34 . 


$$
\left\{\begin{array}{l}
\cos \varphi_{30}=\cos \left(\varphi^{*} \hat{M}\right) \\
\sin \varphi_{30}=\sin \left(\varphi^{*} \hat{M}\right)
\end{array}\right.
$$

$$
\varphi_{30}=\varphi^{*} \hat{M}
$$

\section{THE TRANSITION FROM $2 R$ SYSTEM TO 3R SYSTEM}

In Figure 6 is illustrated spatial kinematic chain.

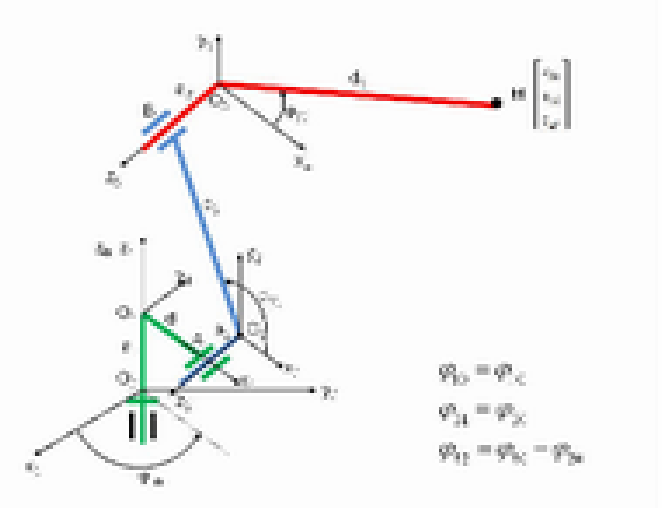

Fig. 6. Geometro spatial kinematic Diagram of a modern structures 3R (anthropomorphic)

The will make the transition from flat to the spatial movement.

Dimensions plane $\mathrm{x}_{2} \mathrm{Oy}_{2}$ will project to the axs zOp. So the length in the vertical axis flat $\mathrm{Oy}$ will be projected on the vertical axis spatial $\mathrm{Oz}$ by adding constant $\mathrm{a}_{1}$, and the length of the horizontal axis flat Ox will be projected on the horizontal spatial axis O $\rho$ by adding constant $\mathrm{d}_{1}$, in accordance with the data relations system (35).

$$
\left\{\begin{array}{l}
\rho_{M^{\prime}}=d_{1}+x_{M}^{P} \\
z_{M}=a_{1}+y_{M}^{P}
\end{array}\right.
$$

Point projections $\mathrm{M}$ on flat axs shall be marked with the index higher $\mathrm{P}$ (plan), in order to detect the presence of appropriate spatial axs.
Due to the fact that the plan of vertical projection axis is moved away from $O \rho$ with a distance constant $a_{2}+a_{3}$, (the plan of vertically work is not projected directly on the axis $O \rho$, but on an axis parallel to it away with a length $\mathrm{a}_{2}+\mathrm{a}_{3}$ ), projection of point $\mathrm{M}$ on horizontal plane in space does not fall in M' but at the point M" (see figure 8).

Because of this projections of $M$ on the spatial axs $\mathrm{Ox}$ and $\mathrm{Oy}$, will not be those of point $\mathrm{M}^{\prime}$ but of the point $\mathrm{M}^{\prime \prime}$, in accordance with the data relations system (36).

$$
\left\{\begin{array}{l}
x_{M}=\rho_{M^{\prime}} \cdot \cos \varphi_{10}+\left(a_{2}+a_{3}\right) \cdot \cos \left(\varphi_{10}+\frac{\pi}{2}\right) \\
y_{M}=\rho_{M^{\prime}} \cdot \sin \varphi_{10}+\left(a_{2}+a_{3}\right) \cdot \sin \left(\varphi_{10}+\frac{\pi}{2}\right)
\end{array}\right.
$$

We would like to eliminate the angle of $90 \mathrm{deg}$ of our relations (36), which have had an important role in the understanding explanatory phenomenon, in order to see how to write the equations for switching from the axs plane in the space, being here (in the horizontal plane in space) comes to a rotation, whose relationships are not to be retained automatically, but deducted logic, that was the reason for which we pass immediately from the system determined logic (36) convenient system (37), which would have been obtained from (36) by eliminating angle of $90 \mathrm{deg}$ from the trigonometric relations.

$\left\{\begin{array}{l}x_{M}=\rho_{M^{\prime}} \cdot \cos \varphi_{10}-\left(a_{2}+a_{3}\right) \cdot \sin \varphi_{10} \\ y_{M}=\rho_{M^{\prime}} \cdot \sin \varphi_{10}+\left(a_{2}+a_{3}\right) \cdot \cos \varphi_{10}\end{array}\right.$

Perhaps it may seem a little difficult method used, but compared to the methods matrix space, it is extremely simple and straightforward, helping to transform spatial movement in a single flat surface, much easier to understand and studied. The system (38) centralizes all relationships move from flat to the 
spatial movement.

$\left\{\begin{array}{l}x_{M}=\left(d_{1}+x_{M}^{P}\right) \cdot \cos \varphi_{10}-\left(a_{2}+a_{3}\right) \cdot \sin \varphi_{10} \\ y_{M}=\left(d_{1}+x_{M}^{P}\right) \sin \varphi_{10}+\left(a_{2}+a_{3}\right) \cdot \cos \varphi_{10} \\ z_{M}=a_{1}+y_{M}^{P}\end{array}\right.$

By replacing in (38) the values of $X_{M}^{P}$ and $y_{M}^{P}$ obtain the equations absolute space (system 39).

$$
\left\{\begin{array}{l}
x_{M}=\left(d_{1}+d_{2} \cdot \cos \varphi_{20}+d_{3} \cdot \cos \varphi_{30}\right) \\
\cdot \cos \varphi_{10}-\left(a_{2}+a_{3}\right) \cdot \sin \varphi_{10} \\
y_{M}=\left(d_{1}+d_{2} \cdot \cos \varphi_{20}+d_{3} \cdot \cos \varphi_{30}\right) \\
\cdot \sin \varphi_{10}+\left(a_{2}+a_{3}\right) \cdot \cos \varphi_{10} \\
z_{M}=a_{1}+d_{2} \cdot \sin \varphi_{20}+d_{3} \cdot \sin \varphi_{30}
\end{array}\right.
$$

For the purpose of determining easier the velocities and acceleration in the system (38), it is noted $a_{2}+a_{3}$ with , so that (38) achieve the stature (40) simplified scheme.

$$
\left\{\begin{array}{l}
x_{M}=\left(d_{1}+x_{M}^{P}\right) \cdot \cos \varphi_{10}-a \cdot \sin \varphi_{10} \\
y_{M}=\left(d_{1}+x_{M}^{P}\right) \cdot \sin \varphi_{10}+a \cdot \cos \varphi_{10} \\
z_{M}=a_{1}+y_{M}^{P}
\end{array}\right.
$$

It is derived as a function of time the system of spatial positions (40) and obtains the system spatial of velocities (41).

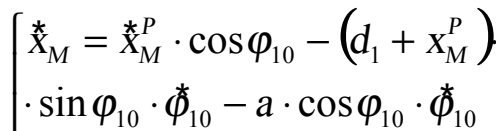

$$
\begin{aligned}
& \left\{\begin{array}{l}
\stackrel{\star}{y}_{M}=\stackrel{*}{x}_{M}^{P} \cdot \sin \varphi_{10}+\left(d_{1}+x_{M}^{P}\right) \\
\cdot \cos \varphi_{10} \cdot \overleftarrow{\varphi}_{10}^{*}-a \cdot \sin \varphi_{10} \cdot \hat{\varphi}_{10}
\end{array}\right. \\
& \stackrel{\star}{Z}_{M}=\grave{y}_{M}^{P}
\end{aligned}
$$

Is derived as a function of time the spatial velocities system (41) and obtains the spatial system of accelerations (42), which is restricted to the form (43).

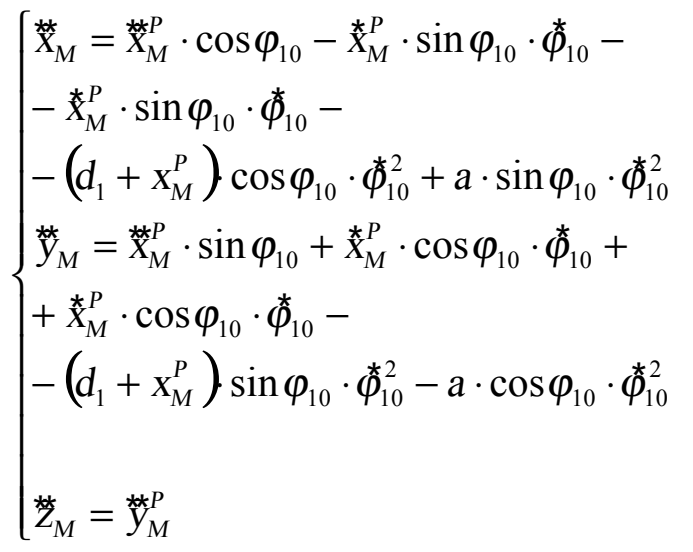

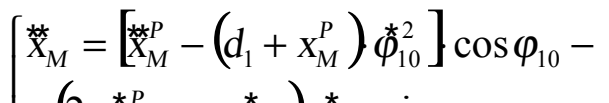

$$
\begin{aligned}
& -\left(2 \cdot \hat{x}_{M}^{P}-a \cdot \hat{\varphi}_{10}\right) \hat{\varphi}_{10}^{*} \cdot \sin \varphi_{10}
\end{aligned}
$$

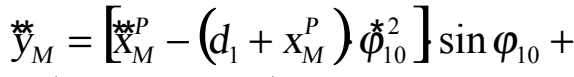

$$
\begin{aligned}
& +\left(2 \cdot \hat{x}_{M}^{P}-a \cdot \dot{\varphi}_{10}\right) \tilde{\varphi}_{10}^{*} \cdot \cos \varphi_{10} \\
& { }_{Z_{M}}={ }^{* y_{M} P}
\end{aligned}
$$

The system spatial of velocities (41) is restricted to the form (44), which by using numbers awarded $\mathrm{u}$ and $\mathrm{v}$ shall be simplified and be written in the form (45). And the system of accelerations (43) can be restricted to form (46), with notations $\mathrm{w}, \mathrm{t}$. 


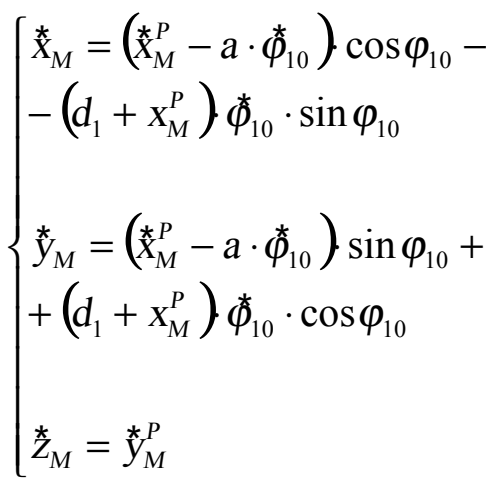

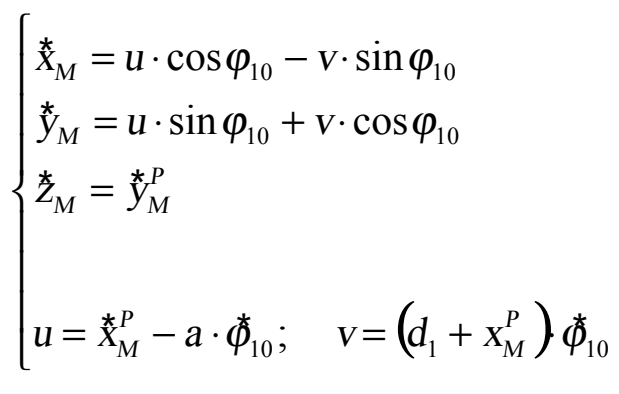

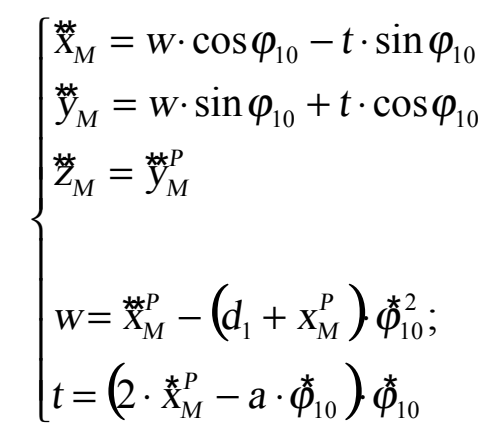

Further, it will present positions, speeds, and spatial accelerations, written all restricted within the framework of the system (47).

$$
\begin{aligned}
& \text { Positions : } \\
& x_{M}=s \cdot \cos \varphi_{10}-a \cdot \sin \varphi_{10} \\
& y_{M}=s \cdot \sin \varphi_{10}+a \cdot \cos \varphi_{10} \\
& z_{M}=a_{1}+y_{M}^{P} \\
& \text { cu } s=d_{1}+x_{M}^{P} ; \quad a=a_{2}+a_{3} \\
& \text { Velocities: } \\
& \vec{X}_{M}=u \cdot \cos \varphi_{10}-v \cdot \sin \varphi_{10} \\
& {\stackrel{*}{y_{M}}}=u \cdot \sin \varphi_{10}+v \cdot \cos \varphi_{10} \\
& {\stackrel{\star}{Z_{M}}}^{*} \stackrel{\star *}{y}_{M}^{P}
\end{aligned}
$$

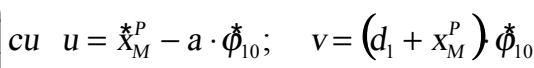

$$
\begin{aligned}
& \text { Accelerations : } \\
& \text { 箱 }_{M}=w \cdot \cos \varphi_{10}-t \cdot \sin \varphi_{10} \\
& y_{M}=w \cdot \sin \varphi_{10}+t \cdot \cos \varphi_{10} \\
& \mathrm{z}_{M}=\mathrm{y}_{M} \\
& \text { with: } \\
& W=\text { 䀅P }_{M}-\left(d_{1}+x_{M}^{P}\right) \cdot \overleftarrow{\phi}_{10}^{2} \text {; } \\
& t=\left(2 \cdot \vec{x}_{M}^{P}-a \cdot \overleftarrow{\phi}_{10}\right) \cdot \overleftarrow{\phi}_{10}
\end{aligned}
$$

Vector module position point spatial end effector $M$, in cartesian space-fixed system is given by the relation (48).

$$
\begin{aligned}
& r_{M}=\sqrt{x_{M}^{2}+y_{M}^{2}+z_{M}^{2}}= \\
& =\sqrt{s^{2}+a^{2}+\left(a_{1}+y_{M}^{P}\right)^{2}}
\end{aligned}
$$

Vector module absolute velocity point spatial end effector $M$, in cartesian space-fixed system is given by the relation (49).

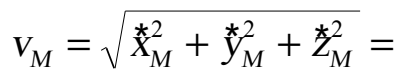

$$
\begin{aligned}
& =\sqrt{u^{2}+v^{2}+\star_{y}^{*} P_{M}^{2}}
\end{aligned}
$$

Vector module absolute acceleration point spatial end effector $M$, in cartesian space-fixed system is given by the relation (50). 


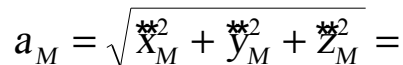

$$
\begin{aligned}
& =\sqrt{w^{2}+t^{2}+{ }^{* y_{M}}{ }^{2}}
\end{aligned}
$$

In the system (51) provide a review of the three parameters space of absolute point $\mathrm{M}$ : absolute position, absolute velocity, acceleration absolute.

$$
\begin{aligned}
& \left\{\begin{array}{l}
r_{M}=\sqrt{x_{M}^{2}+y_{M}^{2}+z_{M}^{2}}= \\
=\sqrt{s^{2}+a^{2}+\left(a_{1}+y_{M}^{P}\right)^{2}}
\end{array}\right.
\end{aligned}
$$

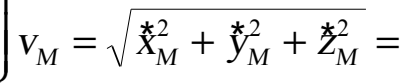

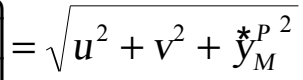

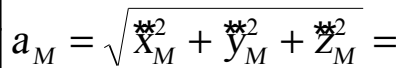

$$
\begin{aligned}
& =\sqrt{w^{2}+t^{2}+\text { 火火p }_{M}^{2}}
\end{aligned}
$$

\section{DISCUSSION}

The method proposed in this work has the advantage of greatly simplify day-to-systems design calculations to mechatronics and robotic systems. Win time, saves work, it's possible to work out a direct synthesis easier serial systems, without it being necessary experimental testing. For teaching method is a total simplification of the design, and thus increasing the understanding of this phenomenon.

If spatial 3R method makes it much easier proposed calculations, the method $2 \mathrm{R}$ make it drastically.

Moving mechanical structures are used increasingly in almost all vital sectors of humanity. The robots are able to process integrated circuits sizes micro and nano, on which the man they can be seen even with electron microscopy. Dyeing parts in toxic environments, working in chemical and radioactive environments, or at depths and pressures at the bottom of huge oceans, or even cosmic space conquest and visiting exoplanets, are now possible, and were turned into from the dream in reality, because mechanical platforms sequential gearbox.

The man will be able to carry out its mission supreme, conquer-or of new galaxies, because mechanical systems sequential gear-box.

Robots were developed and diversified, different aspects, but to-day, they start to be directed on two major categories: systems serial and parallel systems.

Parallel systems are more solid, but more difficult to designed and handled, which serial systems were those which have developed the most.

Serial systems and they have different constructive diagrams, but over the last 30 years have been channelled on anthropomorphic structures.

These structures are made up of simple components and couplers for rotation.

Their great advantage is fast movements, good dynamics, a high accuracy, a construction of simple modules STAS, economy of materials, low cost, and high reliability.

One disadvantage of less accurate has been removed because of stepper motors.

Compared with parallel systems (more solid but more cumber-some) serial systems may pose, and the disadvantage of stability something lower.

This disadvantage begins to be exceeded today in smart mode, through the construction of serial systems made up of elements doubled (in parallel).

This last invention, will lead to the strengthening of serial systems, and to their consolidation like the indisputable 
leader in diversity of mechatronics (Dong, 2013) and robotic systems (Tamura, 2013).

This work starts from a main idea, to study these systems on a single model, $3 \mathrm{R}$, which has finally main movements lying on a single plane model, $2 \mathrm{R}$.

The most commonly used serial structures over the last 20 or 30 years are those of type 3R, 4R, 5R, 6R, having as constituents essential basic kinematic chain 3R, robot anthropomorphic (RRR), where main rotation around a vertical axis, causes the construction.

There are then a basic kinematic chain which has two revolutions 'bokeh' (two actuators, i.e. two motors) who work permanently in one plane, and immediately after main support which supports and rotates vertically complete assembly.

This basic structure $3 \mathrm{R}$, a meet me at all robots serial manufactured on the principle of rotations. Vertical Bracket is was the same, but the drive train as follows, with the two turns situated in a plane can be positioned vertically (most often; the robots anthropomorphic), or horizontally.

It can thus passes from the study spatial movement, which is more difficult, to the study motion plane, basic movement, for all the robots and fillers serial movements of rotation.

Moving flat, horizontal or vertical, shall be undertaken far more easily than the spatial integration with the convenience simple in the space of which it is part.

The basic structure existing in a few serial platforms of rotation, these being the most generalized (more widespread) at the present time (Jin, 2013). On this basic model (3R) have developed further robots 6R today (rely only on revolutions using actuator that only electric motors, compact); they have a hardness greater penetration while maintaining the flexibility and models $3 \mathrm{R}, 4 \mathrm{R}$ and $5 \mathrm{R}$
(Ho, 2013).

Almost all major companies come today with models 6R (which they improve continuously).

Why they have imposed today these models of robots (after tens of years of diversity was the word of order); may and of the need for standardization (Martinez, 2013), or to find a common solution, after a huge portion (however are not yet the only robots use of category serial, but they also have the widest spread) (Park, 2013).

The six turns (full elimination of translations, who bring many disadvantages due to coupler $T$ itself) are operating easier, faster, with higher yield, more reliable, more compact and more secure; basic revolutions, remain all first three, the other three turns (additional) having the role of position may well end device, the end effector. Results and that the baseline study (required) it is still not for a $3 R$.

This can be seen in the latest models of the various firms producing robots (Kawasaki, Romat, Fanuc, Motoman, Kuka, etc). And the structures used inside cells sequential gearbox are constructed generally in a similar way.

Starting from the basic 3R platform may be studied by addition any other scheme, n-R modern (Vázquez, 2013).

The platform (system), has three degree of mobility, which can be realized by three actuators (electric motors). First electric motor drives your whole system in a rotating around a vertical spindle $\mathrm{O}_{0} \mathrm{z}_{0}$. Engine (actuator) number 1 (Aldana, 2013), is mounted on the fixed (frame, 0) and causes mobile element 1 in a rotating around a vertical axis (Wen, 2012).

\section{BENEFITS}

If spatial 3R method makes it much easier proposed calculations, the method $2 \mathrm{R}$ makes calculations much easier and 
faster.

\section{CONCLUSIONS}

The study of spatial systems by decomposing them into plane movements has great advantages relative to understanding phenomena and how writing the equations of motion (much simplified in plan than in space).

The method is original, fast and complete. This article presents just a few aspects of it. Full method includes: building, structure, geometry, kinematics (direct and inverse), calculation of forces, balancing, calculation of forces to a balanced structure, dynamics, dynamic kinematics, transition structures from $2 \mathrm{R}$ to $3 \mathrm{R}$ and vice versa.

\section{REFERENCES}

ALDANA N. D., TRUJILLO C. L., GUARNIZO J. G., Active and reactive power flow regulation for a grid connected vsc based on fuzzy controllers, Revista Facultad de Ingeniería, No. 66 (2013), pp. 118-130.

CAO, W., DING, H., Bin $\mathrm{Zi}$ and Ziming Chen (2013). New Structural Representation and Digital-Analysis Platform for Symmetrical Parallel Mechanisms, International Journal of Advanced Robotic Systems, Sumeet S Aphale (Ed) InTech, DOI: 10.5772/ 56380

U. H., ZHOU, W., LAI, X., and ZHU, S., (2013). An Efficient Inverse Kinematic Algorithm for a PUMA560-Structured Robot Manipulator, IJARS, Jaime Gallardo-Alvarado, Ramon RodriguezCastro (Ed.), ISBN: 1729-8806, InTech, DOI: $10.5772 / 56403$.

HO M. T., RIZAL Y., CHU L.M. (2013). Visual Servoing Tracking Control of a Ball and Plate System: Design, Implementation and Experimental
Validation, International Journal of Advanced Robotic Systems, Stanislav Kovačič (Ed.), ISBN: 1729-8806, InTech, DOI: 10.5772/56525.

MARTÍNEZ H., LAUKKANEN S., MATTILA J. (2013). A New Hybrid Approach for Augmented Reality Maintenance in Scientific Facilities, International Journal of Advanced Robotic Systems, Manuel Ferre, Jouni Mattila, Bruno Siciliano, Pierre Bonnal (Ed.), ISBN: 1729-8806, InTech, DOI: $10.5772 / 56845$

PADULA, F., PERDEREAU, V., (2013). An On-Line Path Planner for Industrial Manipulators, International Journal of Advanced Robotic Systems, Antonio Visioli (Ed.), ISBN: 1729-8806, InTech, DOI: $10.5772 / 55063$.

PARK C. H., PARK D. Il, PARK J. H. (2013). Vibration Control of Flexible Mode for a Beam-Type Substrate Transport Robot, International Journal of Advanced Robotic Systems, Antonio Visioli (Ed.), ISBN: 1729-8806, InTech, DOI: $10.5772 / 56752$.

PERUMAAL, S., JAWAHAR, N., (2013). Automated Trajectory Planner of Industrial Robot for Pick-and-Place Task, IJARS, Antonio Visioli (Ed.), ISBN: 1729-8806, InTech, DOI: 10.5772/53940.

PETRESCU, F.I., PETRESCU, R.V., Cinematics of the 3R Dyad, in journal Engevista, vol. 15, No. 2, (2013), August 2013, ISSN 1415-7314, pp. 118124.

PETRESCU, F.I., PETRESCU, R.V., Kinematics of the Planar Quadrilateral Mechanism, Engevista, vol. 14, No. 3, (2012), December 2012, ISSN 14157314, pp. 345-348.

PETRESCU, F.I., PETRESCU, R.V., Mecatronica - Sisteme Seriale si Paralele, Create Space publisher, USA, 
March 2012, ISBN 978-1-4750-6613-5, 128 pages, Romanian edition.

PETRESCU, F.I, PETRESCU, R.V, Mechanical Systems, Serial and Parallel - Course (in romanian), LULU Publisher, London, UK, February 2011, 124 pages, ISBN 978-1-4466-0039-9, Romanian edition.

PETRESCU, F.I., GRECU, B., COMANESCU, A., PETRESCU, R.V., Some Mechanical Design Elements. In the 3rd International Conference on Computational Mechanics and Virtual Engineering, COMEC 2009, Braşov, October 2009, Edit. UTB, pp. 520-525.

REDDY, P., SHIHABUDHEEN K.V., JACOB, J. Precise Non Linear Modeling of Flexible Link Flexible Joint Manipulator, in International Review on Modelling and Simulations, June 2012, Vol. 5, N. 3B, pp. 13681374.

TABAKOVIĆ, S., ZELJKOVIĆ M., ATALO R. G., ZIVKOVIĆ A., (2013). Program Suite for Conceptual Designing of Parallel MechanismBased Robots and Machine Tools, International Journal of Advanced Robotic Systems, Sumeet S Aphale (Ed.), InTech, DOI: 10.5772/56633.

TAMURA Y.,. TERADA Y., YAMASHITA A. , ASAMA $\mathrm{H}$. (2013). Modelling Behaviour Patterns of Pedestrians for Mobile Robot Trajectory Generation, International Journal of Advanced Robotic Systems, Pablo Gonzalez-De-Santos, Lazaros Nalpantidis, Alejandra Barrera (Ed.), InTech, DOI: 10.5772/56668.

TANG, X., SUN, D., AND SHAO, Z., (2013). The Structure and Dimensional Design of a Reconfigurable PKM, IJARS, Sumeet S Aphale (Ed.), ISBN: 1729-8806, InTech, DOI: 10.5772/54696.
TONG, G., GU, J., XIE, W., (2013). Virtual Entity-Based Rapid Prototype for Design and Simulation of Humanoid Robots, International Journal of Advanced Robotic Systems, Guangming Xie (Ed.), InTech, DOI: 10.5772/55936.

VÁZQUEZ J. A., VELASCO-VILLA M. (2013). Design and Real-Time Control of a 4-DOF Biped Robot, International Journal of Advanced Robotic Systems, Branislav Borovac (Ed.), InTech, DOI: 10.5772/56738.

WANG, K., LUO, M., MEI, T., ZHAO, J., CAO, Y., (2013). Dynamics Analysis of a Three-DOF Planar Serial-Parallel Mechanism for Active Dynamic Balancing with Respect to a Given Trajectory, International Journal of Advanced Robotic Systems, Sumeet S Aphale (Ed.), InTech, DOI: 10.5772/ 54201.

WEN, S., ZHU, J., LI, X., RAD, A., CHEN, X., (2012). End-Point Contact Force Control with Quantitative Feedback Theory for Mobile Robots, IJARS, Houxiang Zhang, Shengyong Chen (Ed.), InTech, DOI: 10.5772/53742. 